05,04

\title{
Высокочастотная магнитная проницаемость однослойных и многослойных нанокомпозитов $\left(\mathrm{Co}_{41} \mathrm{Fe}_{39} \mathrm{~B}_{20}\right)_{x}\left(\mathrm{SiO}_{2}\right)_{100-x}$
}

\author{
(C) О.С. Тарасова ${ }^{1}$, А.В. Ситников ${ }^{1}$, Ю.Е. Калинин ${ }^{1}$, С.Н. Старостенко ${ }^{2}$, А.Б. Грановский ${ }^{2,3, q}$ \\ ${ }^{1}$ Воронежский государственный технический университет, \\ Воронеж, Россия \\ ${ }^{2}$ Институт теоретической и прикладной электродинамики РАН, \\ Москва, Россия \\ ${ }^{3}$ Московский государственный университет им. М.В. Ломоносова, \\ Москва, Россия \\ IE-mail: granov@magn.ru
}

(Поступила в Редакцию 26 мая 2016 г.

В окончательной редакции 8 июня 2016 г.)

Методом ионно-лучевого распыления составной мишени получены тонкие пленки однослойных нанокомпозитов $\left(\mathrm{Co}_{41} \mathrm{Fe}_{39} \mathrm{~B}_{20}\right)_{x}\left(\mathrm{SiO}_{2}\right)_{100-x}$ при $x=30-80$ at.\% и многослойных нанокомпозитов, состоящих из 176 пар слоев состава $\left[\left(\mathrm{Co}_{41} \mathrm{Fe}_{39} \mathrm{~B}_{20}\right)_{60}\left(\mathrm{SiO}_{2}\right)_{40}\right] /\left[\left(\mathrm{Co}_{41} \mathrm{Fe}_{39} \mathrm{~B}_{20}\right)_{60}\left(\mathrm{SiO}_{2}\right)_{40}+\mathrm{O}_{2}\right]$. Концентрационные зависимости магнитной проницаемости однослойных пленок на частоте $50 \mathrm{MHz}$ характеризуются максимумом потерь в окрестности $x=60$ at.\%, тогда как порог перколяции по статической электрической проводимости составляет $x=50$ at.\%. Высокочастотная магнитная проницаемость пленок измерена резонаторным методом в полосе частот $0.1-10 \mathrm{GHz}$. Показано, что при переходе от однослойной пленки к многослойной структуре частота ферромагнитного резонанса смещается от 1.5 до $2.5 \mathrm{GHz}$, при этом мнимая часть магнитной проницаемости достигает значения 200, что предположительно обусловлено подавлением перпендикулярной составляющей магнитной анизотропии.

Исследование выполнено с использованием оборудования, приобретенного за счет Программы развития Московского университета и при финансовой поддержке Российского научного фонда (проект № 16-19-10490). О.С. Тарасова также благодарит за частичную финансовую поддержку РФФИ (проект № 16-32-50207-мол_нр).

\section{1. Введение}

Интерес к высокочастотным свойствам магнитных нанокомпозитов ферромагнитный металл-диэлектрик [1-3] связан прежде всего с их высоким удельным сопротивлением, что позволяет полностью пренебречь потерями на вихревые токи вплоть до частот, превышающих $10 \mathrm{GHz}$, даже для образцов толщиной в несколько микрометров. Кроме того, высокая намагниченность насыщения ферромагнитных металлов, а также возможность управления частотой ферромагнитного резонанса $(Ф М Р)$ за счет изменения магнитной анизотропии в плоскости пленки позволяют считать эти материалы перспективными для применений в СВЧ-диапазоне.

В работе рассмотрена высокочастотная магнитная проницаемость однослойных и многослойных нанокомпозитов аморфный ферромагнитный металл-диэлектрик, в которых в качестве ферромагнетика используется аморфный металл $\mathrm{Co}_{41} \mathrm{Fe}_{39} \mathrm{~B}_{20}$. Хорошо известно, что сплав $\mathrm{Co}_{35} \mathrm{Fe}_{65}$ обладает наивысшей намагниченностью насыщения [4], а при добавлении бора в качестве аморфизатора сплав становится аморфным, при этом его удельное электрическое сопротивление значительно возрастает. Таким образом, использование состава $\mathrm{Co}_{41} \mathrm{Fe}_{39} \mathrm{~B}_{20}$ в композите позволяет в принципе получить материалы с рекордными высокочастотными парамет- рами. Возможным препятствием для этого являются значительная дисперсия локальных осей магнитной анизотропии в плоскости пленок и возникновение перпендикулярной составляющей магнитной анизотропии в процессе напыления при увеличении толщины слоя нанокомпозита. В силу этого необходимы как оптимизация состава композита, так и разработка новых методик подавления перпендикулярной анизотропии. В работе [5] показано, что при циклической подаче реактивных газов в процессе осаждения и образования при этом окисленных прослоек в многослойной структуре можно уменьшить перпендикулярную анизотропию. В настоящей работе этот метод используется для нанесения в едином процессе гетерослойных структур со значительно лучшими высокочастотными магнитными свойствами, чем в случае однослойных нанокомпозитных пленок.

\section{2. Техника эксперимента}

Исследованные нанокомпозиты были получены ионнолучевым распылением составной мишени, представляющей собой металлическую основу сплава $\mathrm{Co}_{41} \mathrm{Fe}_{39} \mathrm{~B}_{20}$, на поверхности которой неравномерно размещено несколько пластин $\mathrm{SiO}_{2}$. Такой метод позволяет в едином технологическом цикле получать композиционные системы 
с непрерывно изменяющимся соотношением металлической и диэлектрической фаз в широких пределах в зависимости от положения подложки относительно мишени $[3,6]$. Ионно-лучевое напыление однослойных композитов проводилось в аргоне на ситалловые подложки размером $60 \times 3 \mathrm{~mm}$, температура которых в процессе напыления не превышала 393 K. Толщина полученных образцов измерялась интерференционным методом и составляла около $1.5 \mu \mathrm{m}$.

Ионно-лучевое напыление многослойной структуры нанокомпозитных пленок проводилось послойно в едином технологическом процессе, но в разной атмосфере газов в напылительной камере. В частности, слои $\left(\mathrm{Co}_{41} \mathrm{Fe}_{39} \mathrm{~B}_{20}\right)_{60}\left(\mathrm{SiO}_{2}\right)_{40}$ напылялись поочередно в течение $47 \mathrm{~s}$ в атмосфере чистого аргона и в течение $15 \mathrm{~s}$ в смеси аргона с кислородом с парциальными давлениями $6 \cdot 10^{-4}$ и $2.4 \cdot 10^{-5}$ Torr соответственно. Толщина бислоя $\left[\left(\mathrm{Co}_{41} \mathrm{Fe}_{39} \mathrm{~B}_{20}\right)_{60}\left(\mathrm{SiO}_{2}\right)_{40}\right] /$ $\left[\left(\mathrm{Co}_{41} \mathrm{Fe}_{39} \mathrm{~B}_{20}\right)_{60}\left(\mathrm{SiO}_{2}\right)_{40}+\mathrm{O}_{2}\right]$ такой структуры не превышала $15 \mathrm{~nm}$. Исследуемый образец состоял из 176 пар слоев, последовательно напыленных на такую же, как и для однослойных пленок, ситалловую подложку.

Элементный состав пленок определялся с помощью локального рентгеноспектрального анализа с использованием энергодисперсионной рентгеновской приставки Oxford INCA Energy-250 на сканирующем электронном микроскопе JEOL JSM-6380 LV. Форма металлических гранул нанокомпозита близка к сферической со средним диаметром не более $2-3 \mathrm{~nm}$.

Магнитостатические измерения выполнены на вибрационном магнитометре LakeShore при комнатной температуре. Измерения комплексной магнитной проницаемости при $50 \mathrm{MHz}$ проводились на измерителе добротности Е4-11. Расчет действительной $\mu^{\prime}$ и мнимой $\mu^{\prime \prime}$ частей магнитной проницаемости осуществлялся по изменению добротности и емкости колебательного контура при внесении исследуемого образца в катушку индуктивности. Измерения комплексной магнитной проницаемости $\mu$ в полосе частот $0.1-10 \mathrm{GHz}$ проводились методом проходного резонатора, в качестве которого использовался отрезок короткозамкнутой коаксиальной линии с изменяемой длиной [7].

\section{3. Обсуждение результатов}

На рис. 1 приведены измеренные концентрационные зависимости удельного электрического сопротивления, а на рис. 2 - магнитной проницаемости при $50 \mathrm{MHz}$ для однослойных нанокомпозитов. Порог перколяции по электрическому сопротивлению составляет $x_{p} \approx 50$ at.\%. Это значение получено согласно апробированной методике [6] по концентрации, при которой пересекаются кривые концентрационных зависимостей электрического сопротивления до и после отжига. Полученное значение хорошо согласуется с порогом перколяции аналогичных

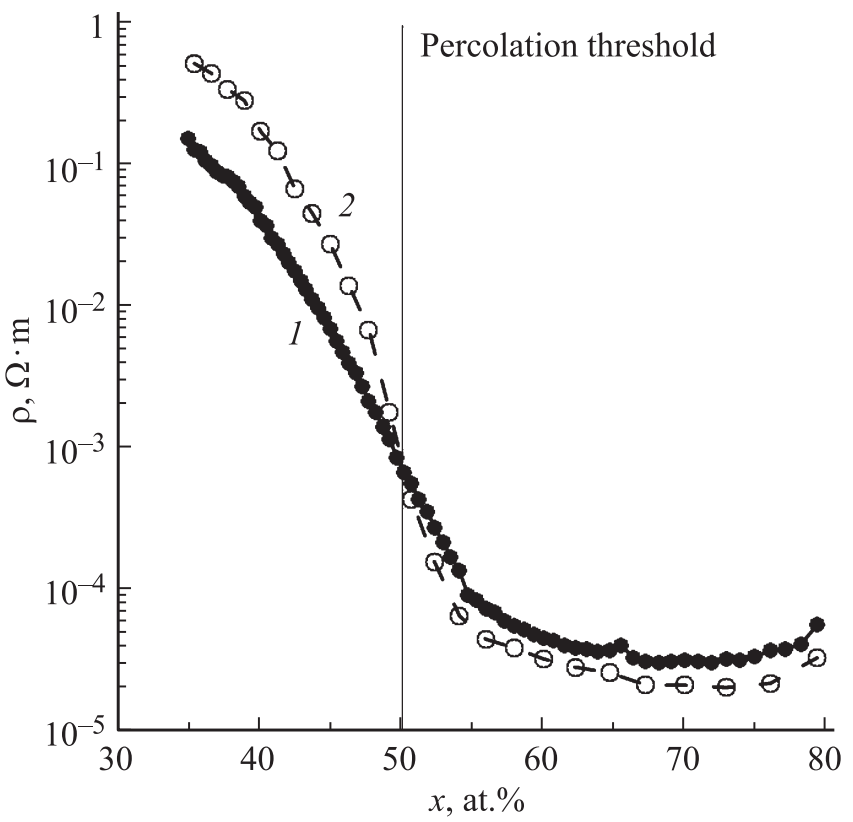

Рис. 1. Зависимости удельного электрического сопротивления $\rho$ однослойных композитов $\left(\mathrm{Co}_{41} \mathrm{Fe}_{39} \mathrm{~B}_{20}\right)_{x}\left(\mathrm{SiO}_{2}\right)_{100-x}$ от содержания металлической фазы $x$ (at.\%) для исходных (1) и отожженных при температуре $350^{\circ} \mathrm{C}$ в течение $30 \mathrm{~min}(2)$ образцов.

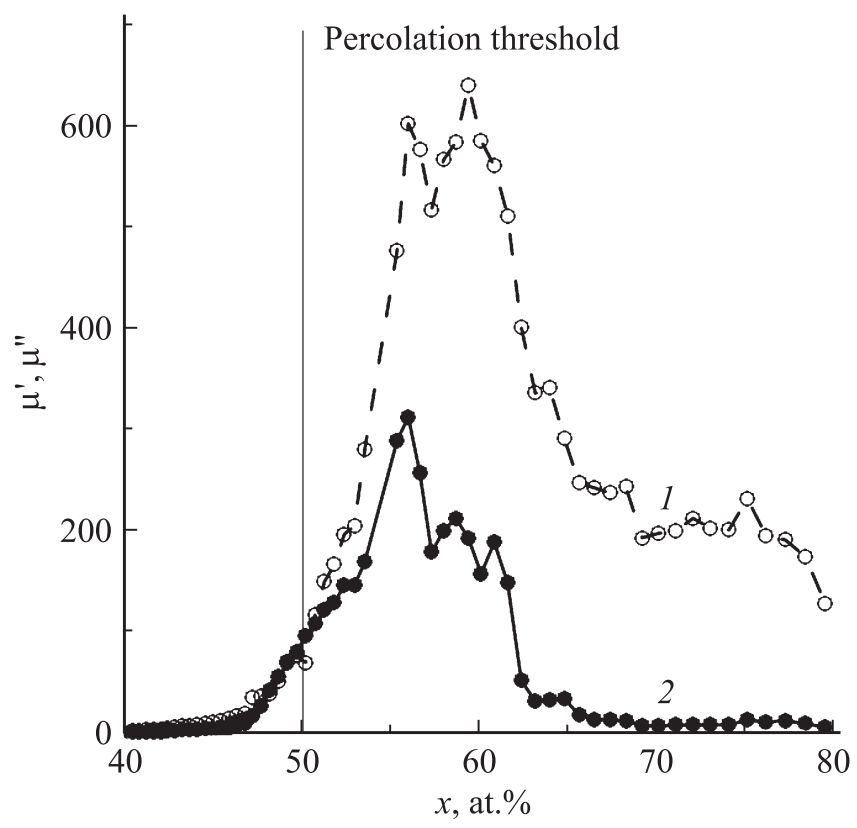

Рис. 2. Зависимости действительной $\mu^{\prime}(1)$ и мнимой $\mu^{\prime \prime}(2)$ частей комплексной магнитной проницаемости композитов $\left(\mathrm{Co}_{41} \mathrm{Fe}_{39} \mathrm{~B}_{20}\right)_{x}\left(\mathrm{SiO}_{2}\right)_{100-x}$ от содержания металлической фазы $x$ (at.\%) при $50 \mathrm{MHz}$.

образцов, определенным по изменению характера проводимости при низких температурах [8]. Вблизи порога перколяции удельное сопротивление образцов достигает $10^{-3} \Omega \cdot \mathrm{m}$, что заведомо позволяет пренебречь потерями на вихревые токи вплоть до $10 \mathrm{GHz}$. 
Магнитная проницаемость при $50 \mathrm{MHz}$ достигает максимума в окрестности $x \approx 60$ at.\%, т. е. в металлической области. Концентрационная зависимость комплексной проницаемости $\mu(x)$ не описывается известными формулами смешения, такими как формула Максвелла-Гарнетта и формула Бруггеманна [2,9]. Это может быть связано с особенностями взаимодействий в магнитных наноструктурах и зависимостью магнитной анизотропии от отношения металл/диэлектрик в составе композита.

Действительно, когда гранулы находятся на большом расстоянии друг от друга, они являются суперпарамагнитными, и их магнитная проницаемость близка к единице. При увеличении концентрации гранул за счет усиливающегося диполь-дипольного взаимодействия и обменного взаимодействия через прослойку диэлектрика происходит переход к ферромагнитному состоянию, что сопровождается увеличением магнитной проницаемости. Переход к ферромагнитному состоянию подтверждается и данными магнитных измерений. Важно, что этот процесс начинается далеко в диэлектрической области, т. е. при $x<x_{p} \approx 50$ at.\%, когда бесконечный перколяционный кластер проводимости еще не образуется. Таким образом, можно считать, что порог перколяции по проводимости не совпадает с порогом перколяции по магнитной проницаемости. При переходе в металлическую область постепенно уменьшается число суперпарамагнитных гранул в мертвых концах перколяционных кластеров и вместо сильно анизотропных путей протекания образуются довольно большие ферромагнитные области, что и сопровождается дальнейшим увеличением проницаемости. В этой области концентраций процесс перемагничивания определяется только вращением вектора намагниченности гранул, поскольку движение доменных границ затруднено из-за морфологии нанокомпозита. Вращение вектора намагниченности - процесс более быстрый, чем движение доменных границ. Это позволяет сохранить высокое значение магнитной проницаемости до более высоких частот, чем для гомогенных ферромагнетиков, которые перемагничиваются посредством движения доменных границ.

Для композитов, расположенных за порогом протекания, возникает нарастающее межгранульное взаимодействие, приводящее к формированию коллективного состояния, магнитные свойства которого начинают доминировать над свойствами отдельных гранул. При этом макроскопические магнитные свойства в существенной степени будут определяться энергией магнитной анизотропии уже самой композитной пленки. Очевидно, что в этой области концентраций (50-70 at.\%) можно ожидать сильной дисперсии локальных осей анизотропии. Это действительно имеет место, так как приложение слабого магнитного поля (10-15 Ое) перпендикулярно оси катушки, которая была использована при измерении магнитной проницаемости на $50 \mathrm{MHz}$, увеличивает магнитную проницаемость практически в 2 раза. Без этого поля величина $\mu^{\prime}$ не превышает 250 (зависимости без приложения дополнительного магнитного поля имеют вид, аналогичный показанному на рис. 2, и поэтому не приводятся). Величина $\mu^{\prime}$ при $x \approx 60$ at.\% достигает максимума, поэтому именно этот состав и выбран как оптимальный для получения многослойных структур. При $x>70$ at.\% практически все гранулы находятся в контакте друг с другом, и магнитная проницаемость уменьшается ввиду увеличения и дисперсии магнитной анизотропии, выхода намагниченности из плоскости пленки, что также подтверждается измерениями петель гистерезиса.

Данные высокочастотных измерений магнитной проницаемости приведены на рис. 3. Из рисунка видно, что для однослойных структур частотная зависимость магнитной проницаемости традиционная, а именно частота ФМР близка к $1.5 \mathrm{GHz}$ при очень широкой резонансной линии. Поскольку частота резонанса однозначно связана с величиной магнитной анизотропии в плоскости пленки, отсюда следует, что в однослойном композите имеется сильная дисперсия анизотропии и, вероятно, возникает перпендикулярная составляющая анизотропии.

Для подавления этих эффектов в литературе предложено несколько способов, а именно напыление многослойных структур из чередующихся слоев металла и диэлектрика или создание обменной анизотропии $[10,11]$. Однако введение немагнитных слоев диэлектрика уменьшает намагниченность насыщения, а использование разных материалов в мультислоях усложняет технологию. Поэтому в настоящей работе выбран подход [5] с послойным напылением из одной и той же составной мишени, но в разной атмосфере газов в камере.

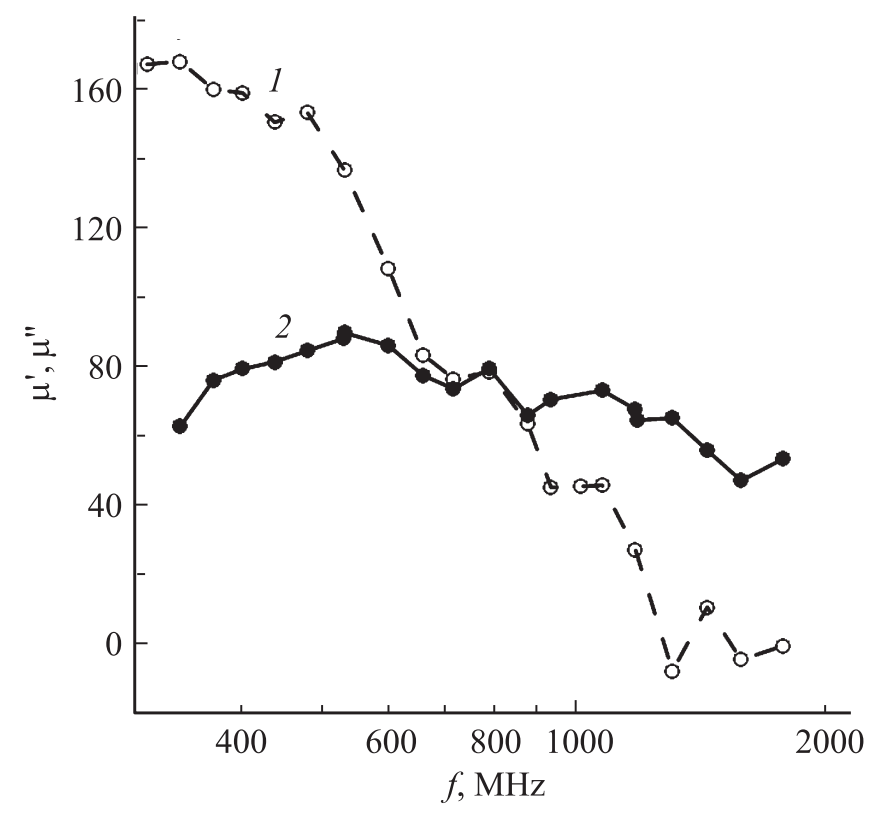

Рис. 3. Зависимости действительной $\mu^{\prime}(1)$ и мнимой $\mu^{\prime \prime}(2)$ частей комплексной магнитной проницаемости от частоты $f$ для однослойного композита $\left(\mathrm{Co}_{41} \mathrm{Fe}_{39} \mathrm{~B}_{20}\right)_{60}\left(\mathrm{SiO}_{2}\right)_{40}$ толщиной $1.5 \mu \mathrm{m}$. 


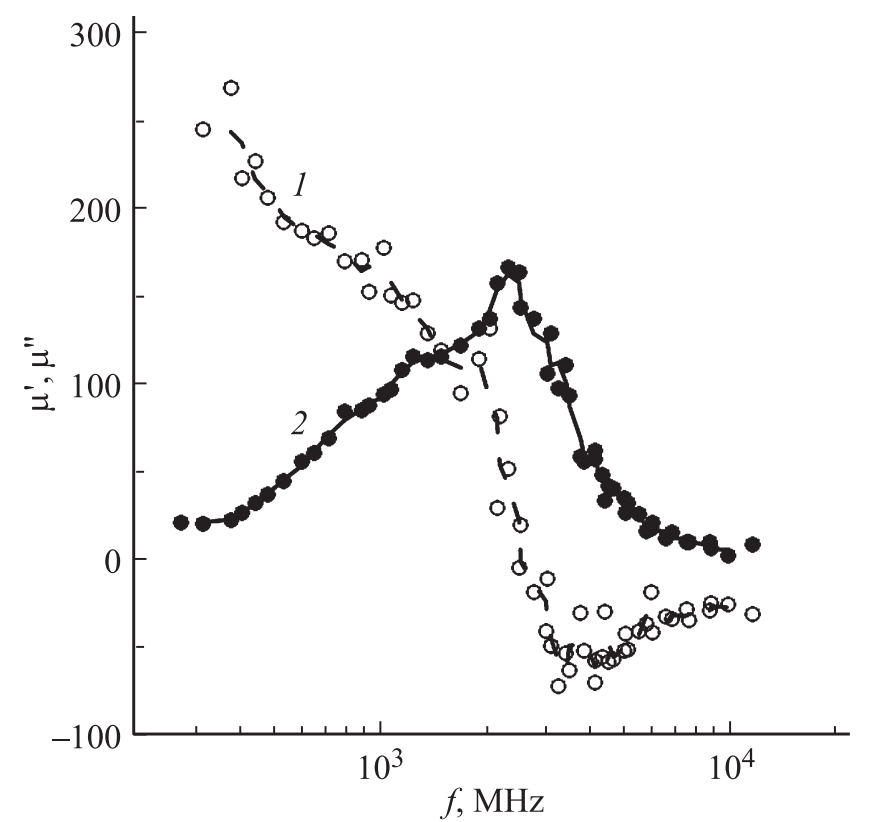

Рис. 4. Зависимости действительной $\mu^{\prime}(1)$ и мнимой $\mu^{\prime \prime}(2)$ частей комплексной магнитной проницаемости от частоты $f$ для гетерогенной структуры из 176 пар слоев $\left[\left(\mathrm{Co}_{41} \mathrm{Fe}_{39} \mathrm{~B}_{20}\right)_{60}\left(\mathrm{SiO}_{2}\right)_{40}\right] /\left[\left(\mathrm{Co}_{41} \mathrm{Fe}_{39} \mathrm{~B}_{20}\right)_{60}\left(\mathrm{SiO}_{2}\right)_{40}+\mathrm{O}_{2}\right]$ с $\quad$ общей толщиной $2.64 \mu \mathrm{m}$.

В результате получена гетерослойная нанокомпозитная структура $\left\{\left[\left(\mathrm{Co}_{41} \mathrm{Fe}_{39} \mathrm{~B}_{20}\right)_{60}\left(\mathrm{SiO}_{2}\right)_{40}\right] /\right.$ $\left.\left[\left(\mathrm{Co}_{41} \mathrm{Fe}_{39} \mathrm{~B}_{20}\right)_{60}\left(\mathrm{SiO}_{2}\right)_{40}+\mathrm{O}_{2}\right]\right\} \times 176$, измеренные магнитные спектры которой приведены на рис. 4.

На основе сравнения рис. 3 и 4 можно сделать заключение о значительном улучшении высокочастотных магнитных свойств гетерослойной структуры по сравнению с однослойной пленкой: частота ФМР увеличивается от 1.5 до $2.5 \mathrm{GHz}$, уменьшается ширина линии ФМР, а на частотах ниже $1 \mathrm{GHz}$ увеличивается действительная часть магнитной проницаемости $\mu^{\prime}$. Это показывает перспективность метода послойного напыления из составной мишени в разной атмосфере газов в камере для повышения высокочастотных магнитных свойств пленок нанокомпозитов.

\section{Список литературы}

[1] N. Vukadinovic. J. Magn. Magn. Mater. 321, 14, 2074 (2009).

[2] R. Ramprasad, P. Zurcher, M. Petras, M. Miller. J. Appl. Phys. 96, 519 (2004).

[3] J.C. Sohn, D.J. Byun, S.H. Lim. Phys. Status Solidi A 201, 8, 1946 (2004).

[4] B.Y. Zong, N.N. Phuoc, Y.P. Wu, P. Ho, F.S. Ma, G.C. Han, Y. Yang, Z.W. Li, S.K. He, Y.H. Wu. ChemElectroChem 2, 11, 1760 (2015).

[5] Н.S.M. Al'Azzavi, А.Б. Грановский, Ю.Е. Калинин, В.А. Макагонов, А.В. Ситников, О.С. Тарасова. ФТТ 58, 5, 910 (2016).
[6] С.А. Гриднев, Ю.Е. Калинин, А.В. Ситников. Нелинейные явления в нано- и микрогетерогенных системах. Бином, M. (2012). $352 \mathrm{c}$.

[7] S.N. Starostenko, K.N. Rozanov. J. Magn. Magn. Mater. 321, 19, 3049 (2009).

[8] Ю.О. Михайловский, В.Н. Прудников, В.В. Рыльков, К.Ю. Черноглазов, А.В. Ситников, Ю.Е. Калинин, А.Б. Грановский. ФТТ 58, 3, 433 (2016).

[9] A.N. Lagarkov, K.N. Rozanov. J. Magn. Magn. Mater. 321, 14, 2082 (2009).

[10] H. Geng, J.Q. Wei, S.J. Niea, Y. Wang, Z.W. Wang, L.S. Wang, Y. Chen, D.L. Peng, F.S. Li, D.S. Xue. Mater. Lett. 92, 346 (2013).

[11] J.H. Chen, D.M. Tang, Y.W. Li, B.S. Zhang, Y. Yang, M. Lu, H.X. Lu. J. Magn. Magn. Mater. 322, 20, 3109 (2010). 